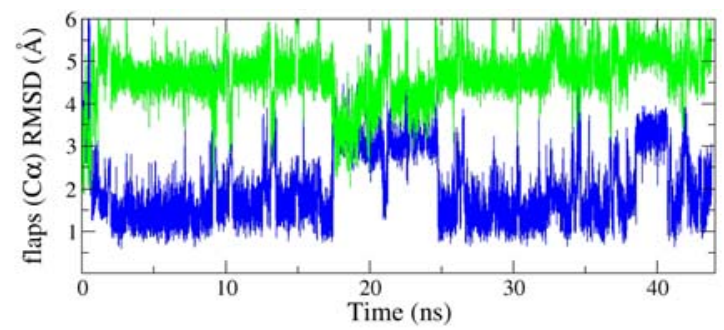

Figure S1. Flaps RMSD for both monomers in the dimer, fit to the 1HHP (semi-open) flaps in blue and the 1TW7 (wide-open) flaps in green. Data is from simulation of the unrestrained dimer in implicit solvent. The initial 1TW7 structure is unstable and the simulated HIVPR adopts the semi-open flap conformation as seen for the wild-type (PDB code 1HHP).
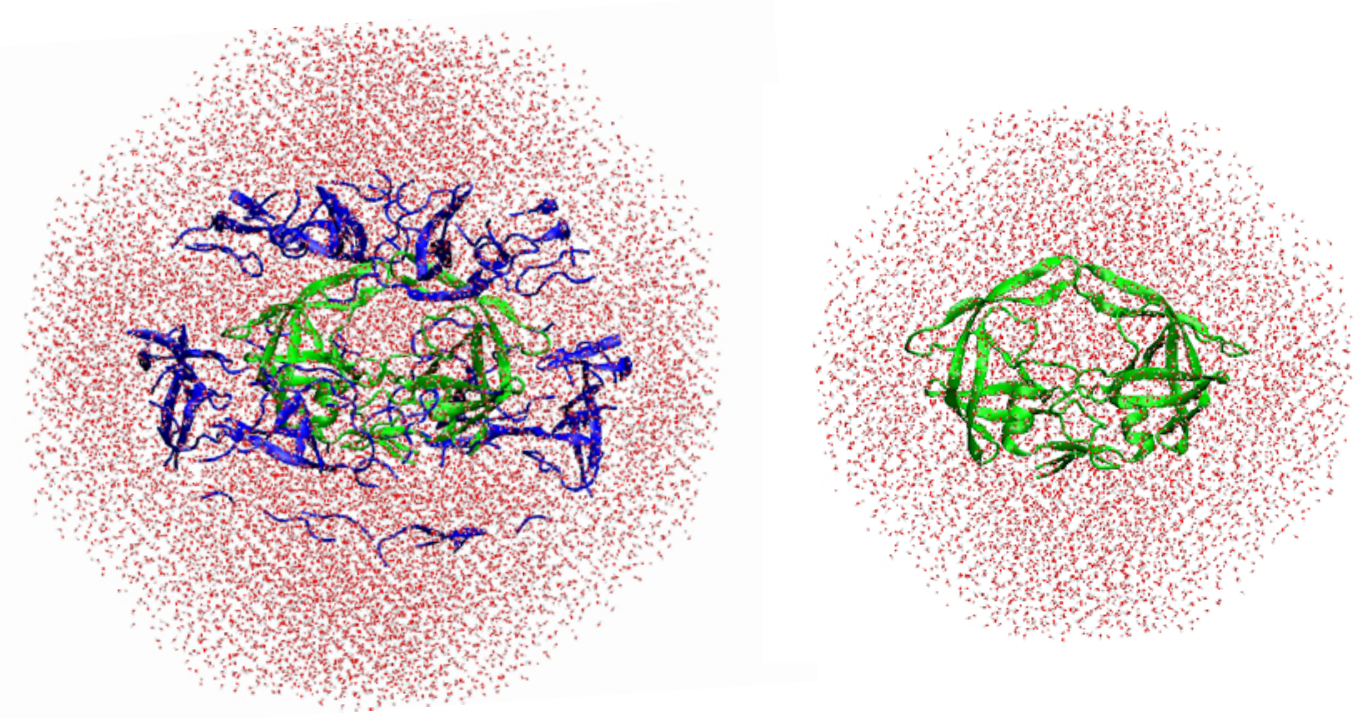

Figure S2. Images of solvated systems used for the simulations. The HIV-PR dimer is shown in a green cartoon diagram and the explicit water is shown in red and white. Left: simulations with crystal packing contacts, with packing residues shown in blue and 20,196 water molecules. Right: The dimer in solution with 8,572 water molecules and no packing contacts.

\title{
Simulation Details:
}

All simulations employed Amber $8^{1}$ and the ff99SB forcefield ${ }^{2}$. Simulations with implicit solvent used a modified generalized Born model $^{3}$ and no cutoff on nonbonded interactions. Simulations with explicit solvent used the TIP3P water model ${ }^{4}$ and truncated octahedral periodic boundary conditions. A cutoff of $8 \AA$ was used on nonbonded interactions, with long-range electrostatic interactions included using the PME method ${ }^{5}$. RMSD of the flap region was calculated using C $\alpha$ atoms in residues 46-55 in both monomers. These protocols are the same as we employed previously for HIV-PR simulations ${ }^{6,7}$.

Positional restraints were applied only to heavy atoms of residues representing crystal packing contacts and not to the central HIV-PR dimer. A force constant of $10 \mathrm{kcal} / \mathrm{mol}-\AA \AA$ was employed.

\section{References}

(1)Case, D. A.; Cheatham, T. E.; Darden, T.; Gohlke, H.; Luo, R.; Merz, K. M.; Onufriev, A.; Simmerling, C.; Wang, B.; Woods, R. J. Journal of Computational Chemistry 2005, 26, 1668-1688.

(2)Hornak, V., Abel, R., Okur, A., Strockbine, B., Roitberg, A. and Simmerling, C. Proteins: Structure, Function and Genetics 2006, in press.

(3)Onufriev, A.; Bashford, D.; Case, D. A. Journal of Physical Chemistry B 2000, 104, 3712-3720.

(4)Jorgensen, W. L.; Chandrasekhar, J.; Madura, J. D.; Impey, R. W.; Klein, M. L. Journal of Chemical Physics 1983, 79, $926-935$.

(5)Darden, T.; York, D.; Pedersen, L. Journal of Chemical Physics 1993, 98, 10089-10092.

(6)Hornak, V.; Okur, A.; Rizzo, R. C.; Simmerling, C. Journal of the American Chemical Society 2006, 128, $2812-2813$.

(7)Hornak, V.; Okur, A.; Rizzo, R. C.; Simmerling, C. Proceedings of the National Academy of Sciences of the United States of America 2006, 103, 915-920. 